\title{
BOUNDARY VALUE PROBLEMS FOR DELAY-DIFFERENTIAL EQUATIONS
}

\author{
BY L. J. GRIMM AND KLAUS SCHMITT ${ }^{1}$
}

Communicated by Wolfgang Wasow, April 29, 1968

1. Introduction. In this note we shall give some sufficient conditions for the existence of solutions of a certain type of boundary value problem (BVP) for delay-differential equations (d.d.e.'s). The conditions given are of two kinds, in Theorem 1 a relationship between the boundary conditions and the size of the interval under consideration implies the existence of solutions; in Theorem 4 the existence of solutions of delay-differential inequalities implies the existence of solutions. A discussion concerning the formulation of BVP's of the type considered here may be found in [1], [2], and [3]; these sources in turn reveal much of the literature concerning such problems.

2. The problem. Let $f$ be a real-valued continuous function defined on $R^{n+m+2} \times I$, where $I$ is the compact interval $[a, b]$. Let $h_{1}(t), \cdots$, $h_{n}(t), g_{1}(t), \cdots, g_{m}(t)$ be nonnegative continuous functions with domain $I$. Assume that $t-g_{i}(t)$ assumes the value $a$ at most a finite number of times as $t$ ranges over $I$ and $i=1, \cdots, m$. Define the real number $c$ by

$$
c=\min \left\{\min _{1 \leqq i \leqq n} \inf _{t \in I}\left(t-h_{i}(t)\right), \min _{1 \leqq j \leqq m} \inf _{t \in I}\left(t-g_{j}(t)\right)\right\}
$$

and let $J=[c, a]$. Let $\phi(t) \in C^{1}(J)$ and let $B$ be any real number; we then seek a function $x(t) \in C(J \cup I) \cap C^{1}(J) \cap C^{1}(I)$ having a piecewise continuous second derivative such that

(1) $x(t)=\phi(t), \quad x^{\prime}(t)=\phi^{\prime}(t), \quad t \in J, \quad x(b)=B, \quad b \leqq b$.

and

(2) $x^{\prime \prime}(t)=f\left(x(t), x\left(t-h_{1}(t)\right), \cdots, x\left(t-h_{n}(t)\right)\right.$,

$$
x^{\prime}(t), x^{\prime}\left(t-g_{1}(t)\right), \cdots, x^{\prime}\left(t-g_{m}(t), t\right)
$$

for $a \leqq t \leqq b$.

In general we must expect that a solution of problem (1)-(2) will have a discontinuous derivative at $t=a$, and therefore the second derivative will in general only be piecewise continuous if the right side of (2) depends on delays in $x^{\prime}$.

1 Research of second author supported by NASA Research Grant NGR-45-003038. 
3. Existence results. Consider now the BVP (1)-(2).

Theorem 1. Let $M>0, N>0$ be given and let $Q=\sup \left\{\left|f\left(x_{1}, \cdots, x_{n+m+2}, t\right)\right|:\left|x_{i}\right| \leqq 2 M, i=1, \cdots, n+1\right.$;

$\left.\left|x_{j}\right| \leqq 2 N, j=n+2, \cdots, n+m+2 ; a \leqq t \leqq b\right\}$.

Then if $b, a<b \leqq b$, is chosen so that

$$
\bar{b}-a \leqq \min \left\{(8 M / Q)^{1 / 2}, 2 N / Q\right\},
$$

$B V P$ (1) - (2) has a solution for any $\phi \in C^{1}(J)$ with $|\phi(t)| \leqq M$, $\left|\phi^{\prime}(t)\right| \leqq N$ and any real number $B,|B| \leqq M$ and

$$
|(\phi(a)-B) /(\bar{b}-a)| \leqq N .
$$

The proof of Theorem 1 may be obtained by means of the SchauderTychonoff Fixed Point Theorem in the following way. We define a mapping $T$ from the Banach space

$$
(B,\|\cdot\|)=\left(C[c, b] \cap C^{1}[c, a] \cap C^{1}[a, b],\|\cdot\|\right),
$$

where

$$
\|x\|=\sup _{c \leqq t \leqq \hat{b}}|x(t)|+\max \left\{\sup _{c \leqq t \leqq a}\left|x^{\prime}(t)\right|, \sup _{a \leqq t \leqq \bar{b}}\left|x^{\prime}(t)\right|\right\},
$$

in to $B$ by

$$
T x(t)=\int_{a}^{\bar{b}} \bar{G}(t ; s) f\left(x(s), \cdots, x^{\prime}(s), \cdots, s\right) d s+l(t)
$$

where

$$
\begin{aligned}
& \bar{G}(t ; s)=G(t ; s), \quad a \leqq t \leqq \bar{b}, \\
& =0, \quad c \leqq t \leqq a, \\
& a \leqq s \leqq b,
\end{aligned}
$$

$G(t ; s)$ is the Green's function with respect to the BVP

$$
x^{\prime \prime}=0, \quad x(a)=0=x(b)
$$

and $l(t)$ is the function

$$
\begin{aligned}
l(t) & =\phi(t), \quad c \leqq t \leqq a, \\
& =\frac{B-\phi(a)}{\bar{b}-a}(t-a)+\phi(a), \quad a \leqq t \leqq \delta .
\end{aligned}
$$

One may then show that $T$ has a fixed point. Fixed points of $T$, however, are solutions of BVP (1) - (2).

The following corollary is important in the proof of the results to follow. 
Corollary 2. Assume there exists a constant $Q$ such that $|f| \leqq Q$ on $R^{n+m+2} \times I$. Then any $B V P(1)-(2)$ has a solution.

Definition. A function $\alpha(t) \in C(J \cup I) \cap C^{1}(J) \cap C^{1}(I)$ having a piecewise continuous second derivative is called a lower solution with respect to BVP (1)-(2) provided

(i) $\alpha(t) \leqq \phi(t), \quad t \in J, \quad \alpha(b) \leqq B$,

(ii) $\alpha^{\prime \prime}(t) \geqq f\left(\alpha(t), \alpha\left(t-h_{1}(t)\right), \cdots, \alpha^{\prime}(t), \alpha^{\prime}\left(t-g_{1}(t)\right), \cdots, t\right)$ for $a \leqq t \leqq b$.

An upper solution $\beta$ of (1)-(2) is defined by reversing the inequalities in (i) and (ii).

Consider now the d.d.e.

$$
x^{\prime \prime}(t)=f\left(x(t), x\left(t-h_{1}(t)\right), \cdots, x\left(t-h_{n}(t)\right), x^{\prime}(t), t\right) .
$$

Lemma 3. Let there exist a constant $Q$ such that $|f| \leqq Q$. Let $\alpha$ and $\beta$ be lower and upper solutions of $B V P(1)-(3)$ with $\alpha(t) \leqq \beta(t)$ for $t \in I$. Furthermore, assume that $f$ is nonincreasing in the second through $(n+1)$ st argument. Then there exists a solution $x(t)$ of $B V P(1)-(3)$ such that $\alpha(t) \leqq x(t) \leqq \beta(t)$ for $t \in I$.

Making use of Lemma 3 we may now obtain results for d.d.e.'s of the form (3) and

$$
x^{\prime \prime}(t)=f\left(x(t), x\left(t-h_{1}(t)\right), \cdots, x\left(t-h_{n}(t)\right), t\right) .
$$

THEOREM 4. Let $f$ be nonincreasing in the second through $(n+1)$ st argument. Then $B V P(1)-(4)$ has a solution if and only if there exist lower and upper solutions $\alpha$ and $\beta$ of (1)-(4) with $\alpha(t) \leqq \beta(t)$ on $I$.

This theorem is very useful in many instances where lower and upper solutions may easily be found. Consider e.g. the following BVP:

$$
\begin{aligned}
& x(t)=\phi(t), \quad c \leqq t \leqq a, \quad x(b)=B, \\
& x^{\prime \prime}(t)=x(t)-x(t-h(t)), \quad a \leqq t \leqq b .
\end{aligned}
$$

Then it is clear that

$$
\beta=\max \left\{\sup _{c \leqq t \leqq a} \phi(t), B\right\} \quad \text { and } \alpha=\min \left\{\inf _{c \leqq t \leqq a} \phi(t), B\right\}
$$

are upper and lower solutions of (5)-(6). Hence there exists a solution $x(t)$ of $(5)-(6)$ such that $\alpha \leqq x(t) \leqq \beta$.

Results similar to Theorem 4 for BVP (1)-(3) may be obtained provided some condition is imposed on $f$ which guarantees a bound 
on the derivative of a solution in terms of a bound on the solution. For example if $f$ satisfies a growth condition

$$
|f| \leqq C_{1}+C_{2}\left|x^{\prime}\right|^{2}
$$

where $C_{1}$ and $C_{2}$ are nonnegative functions of the remaining arguments, then the existence of lower and upper solutions $\alpha$ and $\beta$, $\alpha(t) \leqq \beta(t)$, implies the existence of a solution of BVP (1)-(3).

Proofs of the above results and other existence theorems concerning such BVP's and periodic solutions of d.d.e.'s will appear elsewhere.

\section{REFERENCES}

1. L. E. El'sgol'ts, Introduction to the theory of differential equations with deviating arguments, Holden Day, San Francisco, 1966.

2. A. D. Myshkis and L. E. El'sgol'ts, Some results and problems in the theory of differential equations, Uspehi Mat. Nauk 22 (1967) no. 2, 21-57= Russian Math. Surveys 22 (1967) no. 2, 1967.

3. A. M. Zverkin, G. A. Kemenskii, S. B. Norkin and L. E. El'sgol'ts, Differential equations with a perturbed argument, Uspehi Mat. Nauk 17 (1962) no. 2, 77-164 $=$ Russian Math. Surveys 17 (1962) no. 2, 61-146.

The University OF UtAH 